\title{
ON A THEOREM OF NICOLESCO AND GENERALIZED LAPLACE OPERATORS
}

\author{
MIN-TEH CHENG ${ }^{1}$
}

1. Let $D$ be a domain in the $n$-dimensional Euclidean space and $U$ be a continuous function defined in $D$. Denote by

$$
\mu_{0}(U ; R) \equiv \mu_{0}(U ; P, R)=\frac{1}{\sigma_{n}(R)} \int_{S_{n}(P, R)} U\left(P^{\prime}\right) d \sigma_{P^{\prime}}
$$

the spherical mean of $U$ on the sphere $S_{n}(P, R)$, lying entirely in $D$, with center $P$ and radius $R$, where $\sigma_{n}(R)$ is the surface volume of $S_{n}(P, R)$ and $d \sigma_{P^{\prime}}$ is its volume element. Write $\mu_{k}(U ; R) \equiv \mu_{k}(U ; P, R)$ $=\left(n / R^{n}\right) \int_{0}^{R} t^{n-1} \mu_{k-1}(U ; t) d t$ for $k=1,2, \cdots$.

Blaschke $[1]^{2}$ proved that a necessary and sufficient condition for a continuous function $U(P)$ to be harmonic in $D$ is that

$$
\lim _{R \rightarrow 0} \frac{1}{R^{2}}\left\{\mu_{0}(U ; P, R)-U(P)\right\}=0
$$

holds for every point $P$ in $D$. Nicolesco [4] (also [3, p. 10]) extended Blaschke's theorem as follows.

A necessary and sufficient condition for a continuous function $U(P)$ to be harmonic of order $p$ in $D$ is that, for every point $P$ in $D$,

$$
\lim _{R \rightarrow 0} \frac{1}{R^{2 p}}\left\{\frac{V[\mu, n, p]}{V[1, n, p]}-U(P)\right\}=0,
$$

where $V[\mu, n, p]$ and $V[1, n, p]$ denote the two determinants

$$
\left|\begin{array}{cccc}
\mu_{0}(U ; P, R) & 1 & \cdots & 1 \\
\mu_{1}(U ; P, R) & \frac{n}{n+2} & \cdots & \frac{n}{n+2 p-2} \\
\mu_{2}(U ; P, R) & \frac{n^{2}}{(n+2)^{2}} & \cdots & \frac{n^{2}}{(n+2 p-2)^{2}} \\
\vdots & \vdots & & \vdots \\
\mu_{p-1}(U ; P, R) & \frac{n^{p-1}}{(n+2)^{p-1}} & \cdots & \frac{n^{p-1}}{(n+2 p-2)^{p-1}}
\end{array}\right|
$$

Received by the editors October 31, 1949.

${ }^{1}$ Fellow of The Li Foundation.

${ }^{2}$ Numbers in brackets refer to the references at the end of the paper. 
and

$$
\left|\begin{array}{cccc}
1 & 1 & \cdots & 1 \\
1 & \frac{n}{n+2} & \cdots & \frac{n}{n+2 p-2} \\
1 & \frac{n^{2}}{(n+2)^{2}} & \cdots & \frac{n^{2}}{(n+2 p-2)^{2}} \\
\vdots & \vdots & & \vdots \\
1 & \frac{n^{p-1}}{(n+2)^{p-1}} & \cdots & \frac{n^{p-1}}{(n+2 p-2)^{p-1}}
\end{array}\right|
$$

respectively.

That condition (1.3) in Nicolesco's theorem is necessary follows from his own extension [5] of Gauss' theorem. But the condition is no longer a sufficient one even in the case that $U$ belongs to $C^{\prime}$, that is, that $U$ is continuous together with its first partial derivatives. This can be easily seen from the counter example given below.

2. Let us consider the simple case $n=2$ and $p=2$. In this case, (1.3) reads

$$
\lim _{R \rightarrow 0} \frac{1}{R^{4}}\left\{U(P)+\mu_{0}(U ; R)-2 \mu_{1}(U ; R)\right\}=0,
$$

where

$$
\mu_{0}(U ; R)=\frac{1}{2 \pi} \int_{0}^{2 \pi} U(x+R \cos \theta, y+R \sin \theta) d \theta
$$

and

$$
\mu_{1}(U: R)=\frac{2}{R^{2}} \int_{0}^{R} t \mu_{0}(U ; t) d t
$$

Let us define

$$
U(P) \equiv U(x, y)=\left\{\begin{array}{lr}
x^{2}+y^{2} & (-1<x<1,0 \leqq y<1), \\
x^{2}-y^{2} & (-1<x<1,-1<y<0) .
\end{array}\right.
$$

Obviously $U$ belongs to $C^{\prime}$ throughout in the domain

$$
D: \quad-1<x<1,-1<y<1 \text {. }
$$

Moreover, since $U$ is biharmonic both in the upper half square

$$
-1<x<1, \quad 0<y<1
$$


and in the lower half square

$$
-1<x<1, \quad-1<y<0,
$$

we have only to verify condition (2.1) for

$$
-1<x<1, \quad y=0 .
$$

Let $P=(x, 0)$ be a point on the above segment and $\delta>0$ be chosen so small that, for $0<R \leqq \delta$,

$$
-1<x-R<x+R<1 \text {. }
$$

Then it is easy to see that

$$
\begin{array}{rlrl}
\mu_{0}(U ; P, R)= & \frac{1}{2 \pi} \int_{0}^{2 \pi}(x+R \cos \theta)^{2} d \theta+\frac{1}{2 \pi} \int_{0}^{\pi}(R \sin \theta)^{2} d \theta \\
& -\frac{1}{2 \pi} \int_{\pi}^{2 \pi}(R \sin \theta)^{2} d \theta & \\
= & x^{2}+R^{2} / 2 & (0<R \leqq \delta),
\end{array}
$$

and

$$
\mu_{1}(U ; P, R)=x^{2}+R^{2} / 4 \quad(0<R \leqq \delta) .
$$

Therefore (2.1) holds throughout in $D$. But evidently $U$ is not biharmonic in $D$.

It should be noticed that, in the above example, the relation (2.1) does not hold uniformly on each closed subset of $D$ which contains points of the segment $-1<x<1$ on the $x$-axis. It was pointed out to me by a referee that the following theorem, a slightly weaker form of Nicolesco's theorem, can be proved:

If $U$ is continuous in a domain $D$, and if the relation (2.1) holds uniformly on each closed subset of $D$, then $U(P)$ is biharmonic in $D$.

The proof which the referee sketched to me is based on the fact that, if a function $U$ belongs to $C^{\prime \prime \prime \prime}$, then the relation (2.1) is equivalent to $\Delta^{2} U=0$. Here $\Delta^{2}$ is the ordinary iterated Laplace operator.

We shall also mention here that the original proof given by Nicolesco [4] breaks down at the formula (18) on p. 241 , since a factor $1 / r^{2 p}$ should not be neglected at the left-hand side of this formula.

3. Let us write

$$
\nabla^{2} U=\lim _{R \rightarrow 0} \frac{32}{R^{4}}\left\{\mu_{0}\left(U ; 2^{1 / 2} R\right)-2 \mu_{0}(U ; R)+U(P)\right\}
$$


and

$$
\tilde{\nabla}^{2} U=\lim _{R \rightarrow 0} \frac{192}{R^{4}}\left\{U(P)+\mu_{0}(U ; R)-2 \mu_{1}(U ; R)\right\} .
$$

If $U(x, y)$ belongs to $C^{\prime \prime \prime \prime}$, then

$$
\nabla^{2} U=\tilde{\nabla}^{2} U=\Delta^{2} U(P) .
$$

This can be easily seen in virtue of the following theorem on meanvalues due to Pizzetti [8] (for $n=3$, see Pizzetti [7], and for general $n$-dimensional case, see Nicolesco [5]):

If $U(x, y)$ belongs to $C^{(2 m)}$, then

$$
\begin{aligned}
\mu_{0}(U ; P, R)= & U(P)+\frac{R^{2}}{2^{2}} \Delta U(P)+\frac{R^{4}}{2^{2} 4^{2}} \Delta^{2} U(P)+\cdots \\
& +\frac{R^{2 n-2}}{2^{2} \cdot 4^{2} \cdots(2 n-2)^{2}} \Delta^{2 n-2} U(P) \\
& +\frac{R^{2 n}}{2^{2} \cdot 4^{2} \cdots(2 n)^{2}} \Delta^{2 n} U\left(P^{\prime}\right)
\end{aligned}
$$

where $P^{\prime}$ is a certain point inside the circle $S_{2}(P, R)$.

Both the operators $\nabla^{2}$ and $\tilde{\nabla}^{2}$ can be considered as generalized iterated Laplace operators. However, for the operator $\nabla^{2}$, we can extend Blaschke's theorem as follows:

TheOREM 1. If $U(x, y)$ belongs to $C^{\prime \prime}$ throughout in a domain $D$, and if

$$
\nabla^{2} U(x, y)=0
$$

for every point $(x, y)$ in $D$, then $U(x, y)$ is biharmonic in $D$.

The same example given in $\$ 2$ shows that the hypothesis that $U$ belongs to $C^{\prime \prime}$ cannot be replaced by a weaker one that $U$ belongs to $C^{\prime}$.

We need the following lemma:

Lemma. Let $U$ belong to $C^{\prime \prime}$ in a domain $D$. If

$$
\Delta U(P)=\left(\frac{\partial^{2}}{\partial x^{2}}+\frac{\partial^{2}}{\partial y^{2}}\right) U(x, y)
$$

attains a maximum at a point $Q$ in $D$, and if $\nabla^{2} U$ exists at $Q$, then

$$
\nabla^{2} U(Q) \leqq 0 \text {. }
$$


Proof. Suppose on the contrary that $\nabla^{2} U(Q)>0$, then

$$
\mu_{0}\left(U ; Q, 2^{1 / 2} R\right)-2 \mu_{0}(U ; Q, R)+U(Q)>0
$$

for $0<R<\delta$, say. This implies that

$$
\begin{array}{r}
\frac{4}{\left(2^{1 / 2} R\right)^{2}}\left\{\mu_{0}\left(U ; Q,\left(2^{1 / 2} R\right)-U(Q)\right\}>\frac{4}{R^{2}}\left\{\mu_{0}(U ; Q, R)-U(Q)\right\}\right. \\
(0<R \leqq \delta) .
\end{array}
$$

Thus for $0<R<2^{1 / 2} \delta$, we have

$$
\begin{aligned}
\frac{4}{R^{2}}\left\{\mu_{0}(U ; Q, R)\right. & -U(Q)\} \\
> & \frac{4}{\left(R / 2^{1 / 2}\right)^{2}}\left\{\mu_{0}\left(U ; Q, \frac{R}{2^{1 / 2}}\right)-U(Q)\right\}>\cdots \\
> & \frac{4}{\left(R /\left(2^{1 / 2}\right)^{m}\right)^{2}}\left\{\mu_{0}\left(U ; Q, \frac{R}{\left(2^{1 / 2}\right)^{m}}\right)-U(Q)\right\}>\cdots
\end{aligned}
$$

Since $U$ belongs to $C^{\prime \prime}$, the last member tends to $\Delta U(Q)$ as $m \rightarrow \infty$. Moreover by the mean value theorem we have

$$
\frac{4}{R^{2}}\left\{\mu_{0}(U ; Q, R)-U(Q)\right\}=\Delta U\left(Q^{\prime}\right)>\Delta U(Q)
$$

where $Q^{\prime}$ is a certain point inside the circle $S_{2}(Q, R)$ and can be made arbitrarily near to $Q$ as $\delta$ becomes small. This contradicts the hypothesis that $\Delta U(Q)$ is a maximum.

4. In virtue of the lemma, Theorem 1 can be proved by an argument similar to that in Blaschke [1] (see also Potts [9]). In fact, let $P$ $=\left(x_{0}, y_{0}\right)$ be any point in $D$, and let $S_{2}(P, a)$ be a circle with center $P$ and radius $a$, lying entirely in $D$. Then we have only to show that

$$
\Delta^{2} U(x, y)=0
$$

holds inside $S_{2}(P, a)$.

Let $c>0$ be an arbitrary constant and

$$
\begin{aligned}
h(x, y)=\frac{c}{4}\left\{\frac{1}{12}\left[\left(x-x_{0}\right)^{4}+\left(y-y_{0}\right)^{4}\right]\right. & \\
& \left.-\frac{a^{2}}{4}\left[\left(x-x_{0}\right)^{2}+\left(y-y_{0}\right)^{2}\right]\right\} .
\end{aligned}
$$

Let $V(x, y)$ be a biharmonic function in $S_{2}(P, a)$ subject to the 
boundary condition that

$$
\Delta V(x, y)=\Delta U(x, y)
$$

on $S_{2}(P, a)$. Such a function $V$ can be easily established (see, for example, Nicolesco [6]). Now consider the function

$$
W=U-V+h
$$

which belongs to $C^{\prime \prime}$ inside the circle $S_{2}(P, a)$. It can be easily seen that

$$
\Delta W=\Delta U-\Delta V+\frac{c}{4}\left\{\left(x-x_{0}\right)^{2}+\left(y-y_{0}\right)^{2}-a^{2}\right\}
$$

and

$$
\nabla^{2} W=c
$$

throughout in $S_{2}(P, a)$. Moreover $\Delta W=0$ on the circle $S_{2}(P, a)$. Since $c>0, \Delta W$ must be nonpositive inside $S_{2}(P, a)$. Otherwise $\Delta W$ would attain a positive maximum at a certain point $Q$ inside $S_{2}(P, a)$, which would contradict $(4.2)$ in virtue of the lemma. Thus we have

$$
\Delta U-\Delta V \leqq \frac{c}{4} a^{2}
$$

throughout in $S_{2}(P, a)$. Similarly, by putting $W^{\prime}=V-U+h$, we have also

$$
\Delta V-\Delta U \leqq \frac{c}{4} a^{2} .
$$

But $c$ can be made arbitrarily small; then we have

$$
\Delta U=\Delta V
$$

throughout in $S_{2}(P, a)$. Thus

$$
\Delta^{2} U=\Delta^{2} V=0
$$

in $S_{2}(P, a)$.

5. Another characteristic property of polyharmonic functions has been given by Cioranesco [2]. In case $p=2$ and $n=2$, his theorem is as follows: $D$

If $U(x, y)$ is analytic in a domain $D$ and if for every point $(x, y)$ in 


$$
\lim _{R \rightarrow 0} \frac{1}{R} \int_{0}^{2 \pi}\left[\frac{\partial^{3}}{\partial R^{3}} U(x+R \cos \theta, y+R \sin \theta)\right] d \theta=0,
$$

then $U(x, y)$ is biharmonic in $D$.

The above theorem is an extension of a theorem of Saks [10] concerning harmonic functions. We shall deduce here the following strengthened form of Cioranesco's theorem:

Theorem 2. If $U(x, y)$ belongs to $C^{\prime \prime \prime}$ in a domain $D$, and if (5.1) holds for every point in $D$, then $U(x, y)$ is biharmonic in $D$.

Proof. It can be easily seen in virtue of the law of limit of indetermination that (5.1) implies (3.4). Then Theorem 2 follows from Theorem 1. In fact, let us write

$$
\frac{g(R)}{R^{4}} \equiv \frac{1}{R^{4}}\left\{\mu_{0}\left(U ; 2^{1 / 2} R\right)-2 \mu_{0}(U ; R)+U(P)\right\} .
$$

Then $g(0)=0$. By observing that

$$
\lim _{R \rightarrow 0} \frac{\partial}{\partial R} \mu_{0}(U ; R)=U_{x} \int_{0}^{2 \pi} \cos \theta d \theta+U_{y} \int_{0}^{2 \pi} \sin \theta d \theta=0,
$$

we have $g^{\prime}(0)=0$. Also we have

$$
g^{\prime \prime}(0)=\Delta U(x, y)-\Delta U(x, y)=0 .
$$

Thus by the law of mean,

$$
\frac{g(R)}{R^{4}}=\frac{g^{\prime}(\rho)}{4 \rho^{8}}=\frac{g^{\prime \prime}(\sigma)}{12 \sigma^{2}}=\frac{g^{\prime \prime \prime}(\tau)}{24 \tau} \quad(0<\tau<\rho<\sigma<R) .
$$

Since

$$
\lim _{R \rightarrow 0} \frac{1}{R} g^{\prime \prime \prime}(R)=0
$$

by (5.1), we have

$$
\lim _{R \rightarrow 0} \frac{g(R)}{R^{4}}=0
$$

This proves the theorem.

By the same reasoning we can also prove Theorem 2 directly. Let us consider now the quotient

$$
\frac{f(R)}{R^{8}} \equiv \frac{1}{R^{8}}\left\{R \frac{\partial^{2}}{\partial R^{2}} \mu_{0}(U ; R)-\frac{\partial}{\partial R} \mu_{0}(U ; R)\right\} .
$$


Since $f(0)=0$, then

$$
\frac{f(R)}{R^{3}}=\left[\frac{R \partial^{3} \mu_{0}(U ; R) / \partial R^{3}}{3 R^{2}}\right]_{R=\rho, 0<\rho<R}=\frac{\partial^{3} \mu_{0}(U ; \rho) / \partial \rho^{3}}{3 \rho} .
$$

Thus by (5.1) we have

(5.2) $\lim _{R \rightarrow 0} \frac{f(R)}{R^{3}}=\lim _{R \rightarrow 0} \frac{\partial^{2} \mu_{0}(U ; R) / \partial R^{2}-(1 / R)\left(\partial \mu_{0}(U ; R) / \partial R\right)}{R^{2}}=0$.

On the other hand, by observing that

$$
\begin{aligned}
& \frac{\partial^{2}}{\partial t^{2}} U(x+t \cos \theta, y+t \sin \theta)+\frac{1}{t^{2}} \frac{\partial^{2}}{\partial \theta^{2}} U(x+t \cos \theta, y+t \sin \theta) \\
& =\Delta U(x+t \cos \theta, y+t \sin \theta)-\frac{1}{t} \frac{\partial}{\partial t} U(x+t \cos \theta, y+t \sin \theta),
\end{aligned}
$$

we have the equality

$$
\frac{\partial^{2}}{\partial t^{2}} \mu_{0}(U ; t)=\mu_{0}(f ; t)-\frac{1}{t} \frac{\partial}{\partial t} \mu_{0}(U ; t),
$$

where $f \equiv f(x, y)=\Delta U(x y)$. Thus (5.2) reads

$$
\lim _{R \rightarrow 0} \frac{\mu_{0}(f ; R)-(2 / R)\left(\partial \mu_{0}(U ; R) / \partial R\right)}{R^{2}}=0,
$$

or

(5.4) $\lim _{R \rightarrow 0}\left\{\frac{\mu_{0}(f ; R)-f(x, y)}{R^{2}}-\frac{(2 / R)\left(\partial \mu_{0}(U ; R) / \partial R\right)-f(x, y)}{R^{2}}\right\}=0$.

It can be easily seen that

$$
\lim _{R \rightarrow 0} \frac{2}{R} \frac{\partial}{\partial R} \mu_{0}(U ; R)=f(x, y) .
$$

Thus the second term of (5.4) is again a limit of indetermination. Thus we have

$$
\begin{aligned}
\lim _{R \rightarrow 0} \frac{(2 / R)\left(\partial \mu_{0}(U ; R) / \partial R\right)-f(x, y)}{R^{2}} & \\
=\lim _{R \rightarrow 0} \frac{(2 / \rho)\left(\partial^{2} \mu_{0}(U ; \rho) / \partial \rho^{2}\right)-\left(2 / \rho^{2}\right)\left(\partial \mu_{0}(U ; \rho) / \partial \rho\right)}{2 \rho} \quad(0<\rho=\rho(R)<R) & \quad(0<\rho)
\end{aligned}
$$


which is equal to zero in virtue of (5.2). Then (5.4) reads

$$
\lim _{R \rightarrow 0} \frac{\mu_{0}(f ; R)-f(x, y)}{R^{2}}=0 .
$$

It follows from the theorem of Blaschke that

$$
f(x, y)=\Delta U(x, y)
$$

is harmonic, or $U(x, y)$ is biharmonic.

6. The generalized Laplace operator $\nabla^{2}$ can be defined by (3.1) for any dimension $n \geqq 2$ apart from a constant factor depending on $n$. Theorem 1 and Theorem 2 obviously hold for the general $n$-dimensional case. Theorem 1 also can be extended to polyharmonic functions of order $p$ provided that $\nabla^{p}$, the generalized $p$ th iterated Laplace operator, is properly defined. In this case, $U$ should be supposed of the class $C^{(2 p-2)}$, and Theorem 2 can then also be extended to the polyharmonic case.

In case $n=2$, the generalized 3rd and 4 th iterated Laplace operator should be defined as follows

$$
\begin{aligned}
& \nabla^{3} U=\lim _{R \rightarrow 0} \frac{96}{R^{6}}\left\{\mu_{0}(2 R)-6 \mu_{0}\left(\left(2^{1 / 2}\right) R\right)+8 \mu_{0}(R)-3 U(P)\right\}\left(\mu_{0}(R)=\mu_{0}(U ; P, R)\right), \\
& \nabla^{4} U=\lim _{R \rightarrow 0} \frac{768}{7 \cdot R^{8}}\left\{\mu_{0}\left(2\left(2^{1 / 2}\right) R\right)-14 \mu_{0}(2 R)+56 \mu_{0}\left(2^{1 / 2}\right) R\right) \\
&\left.-64 \mu_{0}(R)+21 U(P)\right\} .
\end{aligned}
$$

The verification of the validity of Theorem 1 and Theorem 2 for $n=2$, $p=3$ and 4 is then obvious.

It would be desirable to obtain the result that Theorem 1 also holds for the operator $\tilde{\nabla}^{2}$; such a result would be closer to Blaschke's result than the present Theorem 1 . However, the difficulty that occurs in our argument is that the lemma used in the proof of Theorem 1 might be invalid for the operator $\tilde{\nabla}^{2}$.

\section{REFERENCES}

1. W. Blaschke, Ein Mittelwertsatz und eine kennzeichnende Eigenschaft des logarithmischen Potentials, Leipziger Berichte vol. 68 (1916) pp. 3-7.

2. N. Cioranesco, Une propriêté caractéristique des fonctions polyharmoniques, Bulletinul Facultății de Sțiinţe din Cernăuti vol. 7 (1933) pp. 105-107.

3. M. Nicolesco, Les fonctions polyharmoniques, Exposés sur la théorie des fonctions, vol. 4, 1936, Paris.

4. - - Sur une proprieté caractéristique des fonctions harmoniques d'ordre p et 
sur lexistence des laplaciens de divers ordres, Buletinul Facultaţii de Sţiinte din Cernăuti vol. 7 (1933) pp. 235-243.

5. - Sur les fonctions de $n$ variables, harmoniques d'ordre $p$, Bull. Soc. Math. France vol. 50 (1932) pp. 129-151.

6. - REsolution effective directe qde uelques problèmes de frontière concernant les fonctions harmoniques et biharmoniques dans le cas des domaines hyperspheriques, Buletinul Facultătii de Sţiinte din Cernauti vol. 9 (1935) pp. 9-15.

7. P. Pizzetti, Sulla media dei valori che una funzione dei punti dello spasio assume alla superficie di una sfera, Rendiconti dei Lincei (5) vol. 18 (1909) pp. 182-185.

8. - Sul significato geometrico del secondo parametro differensiale di una funzione sopra una superficie qualunque, Rendiconti dei Lincei (5) vol. 18 (1909) pp. 309-316.

9. D. H. Potts, A note on the operators of Blaschke and Privaloff, Bull. Amer. Math. Soc. vol. 54 (1948) pp. 782-787.

10. S. Saks, Note on defining properties of harmonic functions, Bull. Amer. Math. Soc. vol. 38 (1932) pp. 380-382.

Princeton University 